\title{
SIS Epidemic Model Birth-and-Death Markov Chain Approach
}

\author{
A.H. Nzokem \\ Correspondence: Department of Mathematics \& Statistics, York University, Toronto \\ Received: February 24, 2021 Accepted: May 21, 2021 Online Published: May 28, 2021 \\ doi:10.5539/ijsp.v10n4p10 \\ URL: https://doi.org/10.5539/ijsp.v10n4p10
}

\begin{abstract}
We are interested in describing the dynamics of the infected size of the SIS Epidemic model using the Birth-Death Markov process. The Susceptible-Infected-Susceptible (SIS) model is defined within a population of constant size $M$; the size is kept constant by replacing each death with a newborn healthy individual. The life span of each individual in the population is modelled by an exponential distribution with parameter $\alpha$; the disease spreads within the population is modelled by a Poisson process with a rate $\lambda_{I} . \lambda_{I}=\beta I\left(1-\frac{I}{M}\right)$ is similar to the instantaneous rate in the logistic population growth model. The analysis is focused on the disease outbreak, where the reproduction number $R=\frac{\beta}{\alpha}$ is greater than one. As methodology, we use both numerical and analytical approaches. The numerical approach shows that the infected size dynamics converge to a stationary stochastic process. And the analytical results determine the distribution of the stationary stochastic process as a normal distribution with mean $\left(1-\frac{1}{R}\right) M$ and Variance $\frac{M}{R}$ when $M$ becomes larger.
\end{abstract}

Keywords: deterministic model, stochastic model, Birth - Death Markov Chain, Irreducible Markov Chain (IMC), Jensen inequality, epidemic model

\section{Introduction}

The Birth and Death Markov Chain is a special class of the continuous stochastic process. The importance of such class arises from two standard processes (Birth process and Death process). The stationary distribution of the Birth and Death process was studied in the literature (Karlin,1975). The findings describe the behavior of the stochastic process in the long run; when the time variable has not to effect on the distribution process. The Susceptible-Infected-Susceptible (SIS) model is one of the simplest and paradigmatic models in mathematical epidemiology. The stochastic version of the SIS model was studied by näsell $(1996,1999)$, who is among the pioneers to report the normal distribution nature of the quasistationary distribution when the reproduction number is greater than one and the population size $(M)$ is larger. The major critic was the methodology. Ovaskainen (2001) argues that the methodology was heuristic. In general, the literature review (Clancy \& Mendy (2011),Wierman \& Marchette (2004)) offers different approximations of the quasi-stationary distribution of the SIS model, due to the difference in methodology or in parametrization. Wierman \& Marchette (2004) consider transmission parameter $(\beta)$ as a function of the population size $(M)$ in their paper.

The paper is structured as follows; in the next section, we will determine the disease dynamics parameters and analyze the infected size through sample and distribution simulations; and the last section will focus on analyzing the probabilistic distribution of the asymptotic infected size.

\section{Epidemic Spreading: Death and Infection Process Modelling}

The SIS model population is divided into susceptible individuals $(S(t))$ and infected individuals $(I(t))$ at each time $t$. The evolution of these quantities is usually described in Epidemiology by the following deterministic differential equations (2.1) $(a)$ :

(a)

$$
\begin{aligned}
\frac{d S}{d t} & =-\frac{\beta}{M} S I+\alpha I & \frac{S}{M} & =\frac{\alpha}{\beta}=\frac{1}{R} \\
\frac{d I}{d t} & =\frac{\beta}{M} S I-\alpha I & \frac{I}{M} & =1-\frac{\alpha}{\beta}=1-\frac{1}{R}
\end{aligned}
$$

The parameters $\beta$ and $\alpha$ are respectively the transmission rate and the rate of death and birth. To have population size $(M=S+I)$ constant over time, each individual who dies is replaced by a susceptible individual.

The threshold value $R=\frac{\beta}{\alpha}$, which is a basic Reproduction Number, determines whether the disease will decline and eventually die out $(0<R<1)$ or the disease will develop to an outbreak or epidemic $(R>1)$; in the special case where $R=1$, the disease stay alive and stable, but there is no outbreak or epidemic.

One of the results of the deterministic differential equations is the equilibrium $(2.1)(b)$ of the system (2.1) (a) in the long 
run with $R>1$. At the equilibrium $(2.1)(b)$, the portion infected is constant $\left(1-\frac{1}{R}\right)$. The deterministic version of the SIS model was introduced by Kermack and McKendrick and has been fully analysed. For some related deterministic work of interest, see Nzokem \& Madras (2020, 2021).

The stochastic version, called the stochastic logistic epidemic model, is modelled as the Birth and Death Markov Chain with the transition probability defines as follows.

$$
P_{I, J}(t, t+h)= \begin{cases}\lambda_{I} h+o_{1}(h) & \text { if } J=I+1 \\ \mu_{I} h+o_{2}(h) & \text { if } J=I-1 \\ 1-\left(\lambda_{I}+\mu_{I}\right) h+o_{3}(h) & \text { if } J=I\end{cases}
$$

with $\lim _{h \rightarrow 0} o_{1}(h)=0 ; \lim _{h \rightarrow 0} o_{2}(h)=0 ; \lim _{h \rightarrow 0} o_{3}(h)=0$

We suppose the transmission rate $\beta=c \theta$ can be written as a product of contact rate $(c)$ and the probability of infection $(\theta)$. The transition probability (2.2) can be fully determined by the following set of rules (Clancy, 2011): (a) each individual gets into contact with another individual after an elapsed time, which follows an exponential distribution with parameter $c$. And if the contact involves a susceptible individual and infected individual, the probability of infection is $\theta$. The transmission rate is $\beta=c \theta$. (b) the infected lifetime is also an exponential distribution with parameter $\alpha$, because of the memoryless property of the lifetime.

For $J=I+1$ in (2.2), all I infected individuals get into contact with another individual according to a Poisson process with parameter $c I$, since $\left(1-\frac{I}{M}\right)$ is the probability to meet susceptible individuals and $\theta$ is the probability of infection, by thinning the Poisson process, we conclude that the contacts between infected and susceptible individuals that will end up with infection follows a Poisson process with parameter $\lambda_{I}=\beta I\left(1-\frac{I}{M}\right)$.

For $(J=I-1)$ in $(2.2)$, the number of infected individuals that becomes susceptible individuals follows a Poisson process with parameter $\mu_{I}=\alpha I$.

\subsection{Infected Size Samples}

Based on the assumptions and parameters developed previously, a MATLAB program with $M$ individuals was created, and the main variables were age, health status, and cumulative elapsed time between events. At the death of each individual, a healthy individual and his life span are automatically replaced in the program code. By controlling the age, we can focus on the infection process over time.

For $R=2$ and $\alpha=0.3$, we have the infected size as a function of cumulative elapsed time between events. Two sample paths are presented in Fig 1 with only one infected initially and with 95\% infected initially.



Figure 1. Deterministic versus Stochastic equilibrium of the infected size

Only one individual is infected initially for the sample in red colour, the infected size grows at an increasing rate before fluctuating around the deterministic equilibrium $M\left(1-\frac{1}{R}\right) .95 \%$ of individuals are infected initially for the sample in blue colour, as shown in Fig 1, the infected size decreases rapidly before fluctuating around the deterministic equilibrium.

The impact of reproduction number $(\mathrm{R})$ on the sample path is considered in Fig 2. In fact, when there is only one infected initially and the reproduction number is greater than 1, the infected size increases rapidly, before fluctuating around deterministic equilibrium $M\left(1-\frac{1}{R}\right)$, straight line in black color in Fig 2. The same pattern is observed when there is $95 \%$ infected initially, the fluctuation follows a rapidly decreasing. 
The stability is observed in Fig 2 for $R=2$ and $R=4$; whereas for $R=1$, the process is unstable, and the disease will eventually die out. In addition, for $R \geq 1$, there is a positive relationship between the infected size and the reproduction number $(R)$. As illustrated in Fig 2, the infected size increases when $R$ increases.



Figure 2. Impact of the Reproduction Number $(R)$ on the equilibrium

\subsection{Infected Size Stationary Distribution}

Based on the parameters developed in (2.2), we have a Birth and Death process on the state space $\{0,1, \ldots, M\}$ with transition rates $(2.3)$ :

$$
\lambda_{k}=\beta\left(\frac{M-k}{M}\right) k \quad(k \rightarrow k+1) \quad \mu_{k}=\alpha k \quad(k \rightarrow k-1)
$$

However, $\lambda_{0}=0$, as shown the following Graph, we have two equivalence classes: $\{1,2, \ldots, \mathrm{M}\}$ and $\{0\}$. The Markov chain is not irreducible, but absorbable.

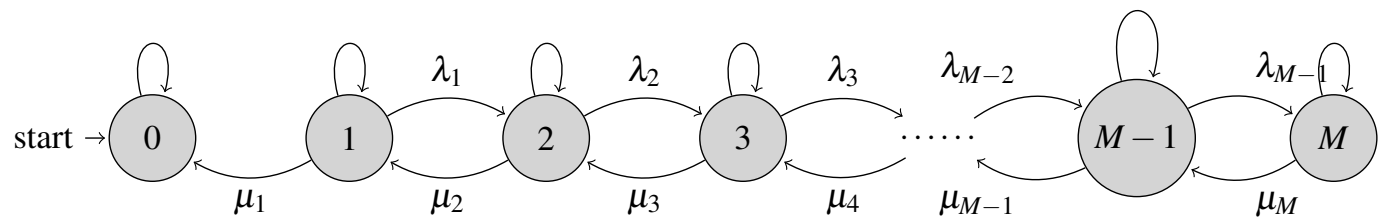

In order to have an irreducible Markov Process, we introduce an external source of disease through a small nonnegative parameter $(\varepsilon>0)$ in the infection transition rate (2.4). We have a new Process $P_{M, \varepsilon}$ on the state space $\{0,1, \ldots, M\}$.

$$
\lambda_{i}^{[\varepsilon]}=\beta \frac{(M-i)}{M} i+\varepsilon \quad(i \rightarrow i+1) \quad \mu_{i}=\alpha i \quad(i \rightarrow i-1)
$$

According to Karlin et al $(1975,1998)$, we define the quantities $(2.5)$ :

$$
\theta_{0}=1 \quad \theta_{i}^{[\varepsilon]}=\frac{\lambda_{0}^{[\varepsilon]} \lambda_{1}^{[\varepsilon]} \cdots \lambda_{i-1}^{[\varepsilon]}}{\mu_{1} \mu_{2} \cdots \mu_{i}} \quad \text { for } i=1, \ldots, M .
$$

Therefore, we have the stationary distribution of $P_{M, \varepsilon}$.

$$
\pi_{M}^{[\varepsilon]}(i)= \begin{cases}\frac{\theta_{0}}{\theta_{0}+\sum_{j=1}^{M} \theta_{j}^{[\varepsilon]}} & i=0 \\ \frac{\theta_{i}^{[\varepsilon]}}{\theta_{0}+\sum_{j=1}^{M} \theta_{j}^{[\varepsilon]}} & (i=1,2, \ldots, M)\end{cases}
$$

In order to have an overview of the shapes of the stationary distribution, we look at four cases with Reproduction Number (R): $R=1, R=1.5, R=2$, and $R=2.5$. For $R=1$, the distribution is mostly concentrated at state 0 , but not only at state 0 as shown in Fig 3a.

When $R$ is greater than 1 , the density seems continuous and symmetrical. For $R=1.5$, the density distribution has a bell-shaped curve and spreads around the mean of 66 infections, as shown in Fig 3b. For $R=2$, as shown in Fig 3c, the distribution is still bell-shaped, but the spreading is centered at 100 infections. 


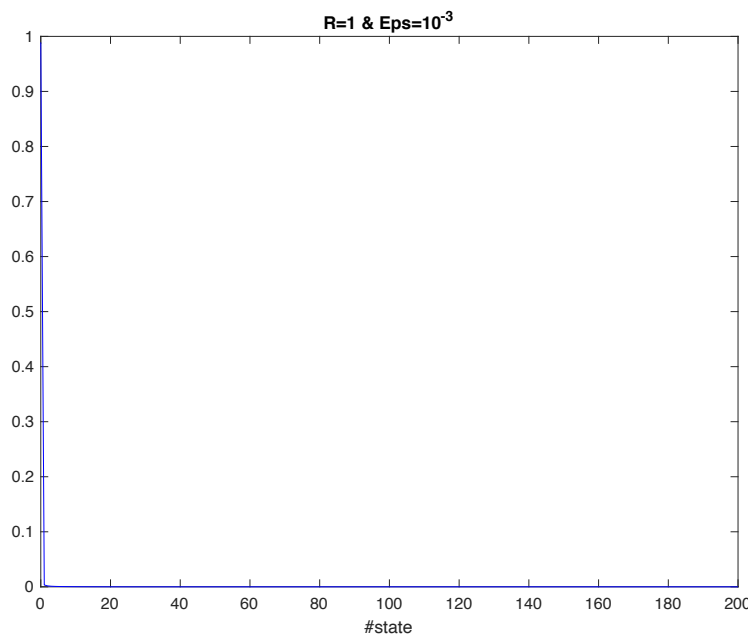

(a) Distribution concentrates at 0



(c) Bell-shaped distribution around 100

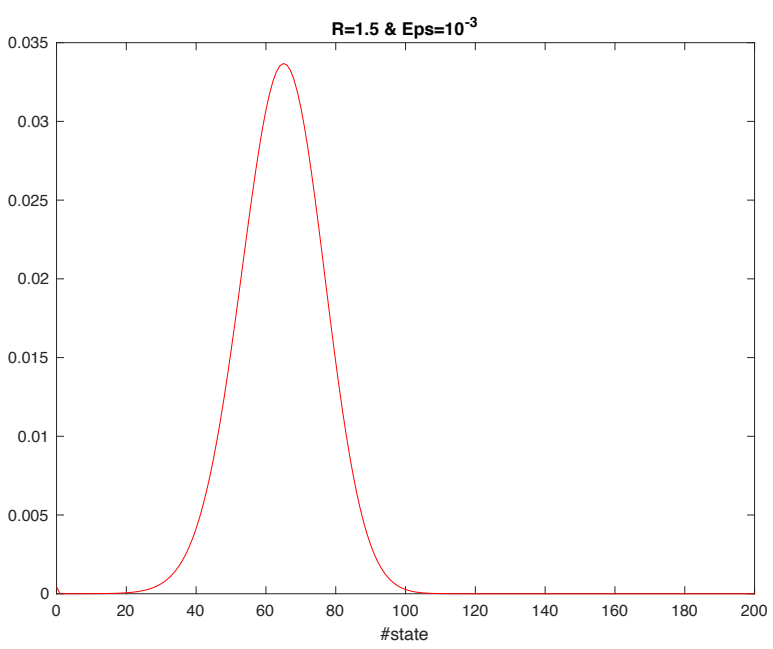

(b) Bell-shaped distribution around 66

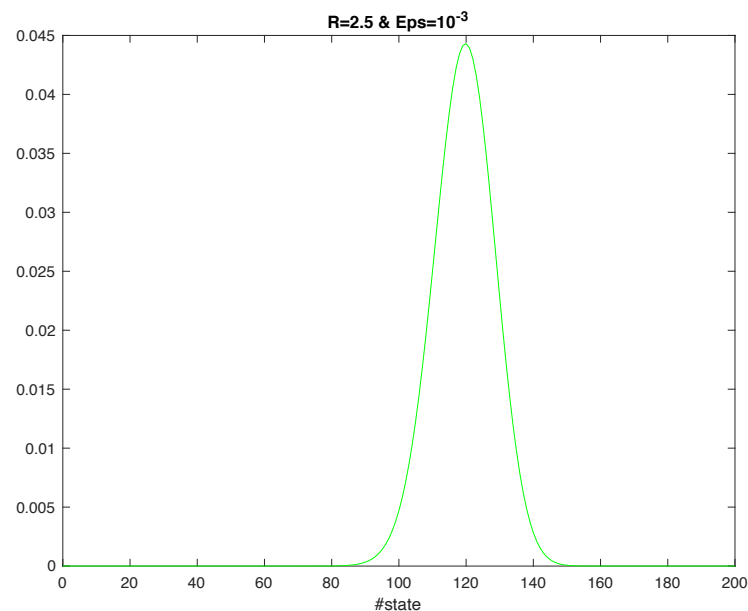

(d) Bell-shaped distribution around 120

Figure 3. Distribution $\left(P_{M, \varepsilon}\right): \varepsilon=10^{-3}, M=100, \alpha=0.3, \beta=R \alpha$

Given the distribution $\left(P_{M, \varepsilon}\right)$, we can look at some measures of central tendency and dispersion. Four statistical indicators (mean, variance, skewness (skew), and kurtosis (Kurt)) are computed to summarize the characteristics of the distribution.

$$
\bar{x}=\sum_{j=0}^{M} \pi_{M}^{[\varepsilon]}(j) j \quad \sigma^{2}=\sum_{j=0}^{M} \pi_{M}^{[\varepsilon]}(j)(j-\bar{x})^{2} \quad \text { Skew }=\sum_{j=0}^{M} \pi_{M}^{[\varepsilon]}(j)\left(\frac{j-\bar{x}}{\sigma}\right)^{3} \quad \text { Kurt }=\sum_{j=0}^{M} \pi_{M}^{[\varepsilon]}(j)\left(\frac{j-\bar{x}}{\sigma}\right)^{4}
$$

As illustrated in Fig 4a, the mean $(\bar{x})$ of infected size is a function of the population size $(M)$. When the population size $(M)$ is small, the mean increases at a growing rate before reaching a stable position as $M$ becomes greater than 100 . At this stage, the mean continues to increase but at a constant rate $\left(1-\frac{1}{R}\right)$. In Fig $4 \mathrm{~b}$, the variance $\left(\sigma^{2}\right)$ is also a function of the population size $(M)$ and is unstable when $M$ is not large $(M<100)$. When $M$ becomes large, the variance reaches a stable phase where the increasing is at a constant rate $\left(\frac{1}{R}\right)$.

As shown in Fig 4, the results of the Skewness and Kurtosis indicators are independent of reproduction number $(R)$.

The Skewness is an indicator of lack of symmetry, that is, both left and right sides of the distribution $\left(P_{M, \varepsilon}\right)$ are unequal with respect to the mean. In Fig 4c, the Skewness (Skew) as a function of $M$ shows that the distribution of the system lacks symmetry when $M$ is not large enough $(M<100)$. When $M$ becomes large enough, the Skewness converges to 0; and the symmetric nature of the distribution appears.

The Kurtosis is a measure of how heavy-tailed or light-tailed the distribution $\left(P_{M, \varepsilon}\right)$ is relative to a normal distribution. In Fig 4d, the Kurtosis(Kurt) as a function of $M$ shows that the SIS model alternates between heavy-tailed and light-tailed before reaching a stable value of 3 , when $M$ becomes large enough. It is important to point out that the normal distribution has kurtosis equal to 3. 


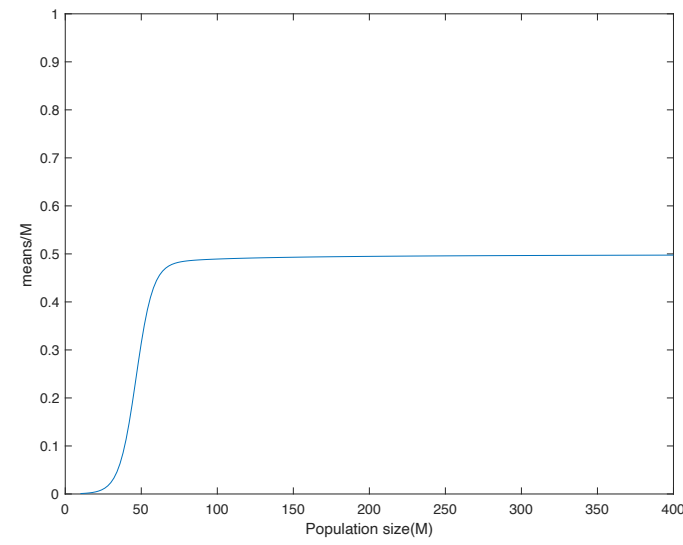

(a) Mean/M converges to $1-\frac{1}{R}$



(c) Skewness converges to 0

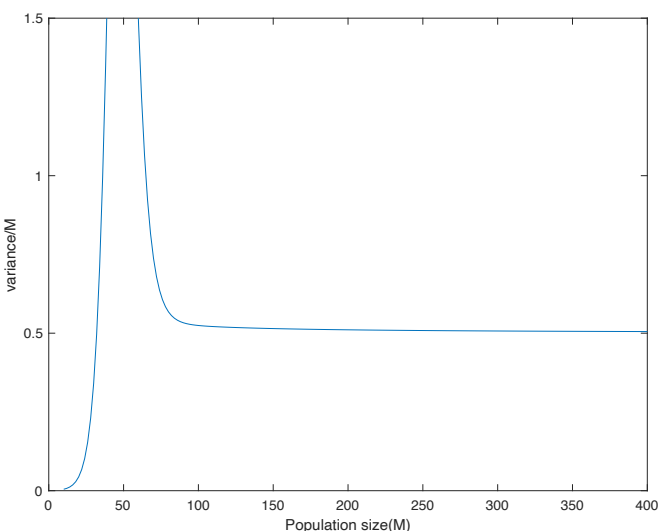

(b) Variance/M converges to $\frac{1}{R}$

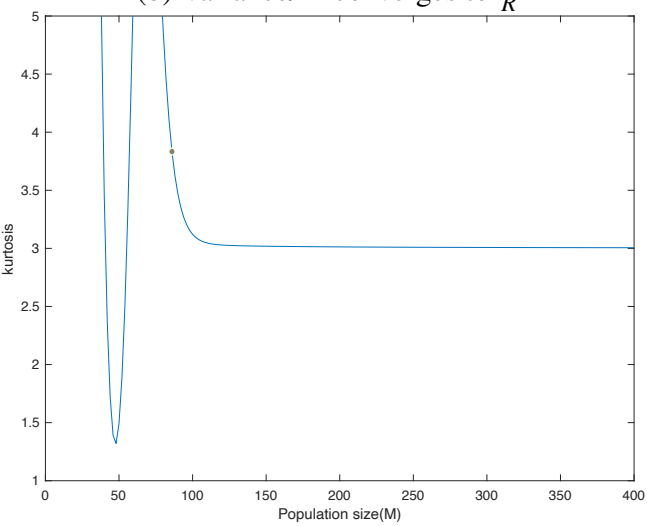

(d) Kurtosis converges to 3

Figure 4. Central and dispersion characteristics of the distribution $\left(P_{M, \varepsilon}\right): R=2, \varepsilon=10^{-4}$ and $\alpha=0.3$

Figs 3 and 4 provide evidence that the infected size follows a normal distribution when the population size(M) reaches a certain threshold.

\section{Infected Size Distribution: Analytical Results}

We revise the continuous-time Markov chain on the state space $\{0,1, \ldots, M-1\}$ with transition rates.

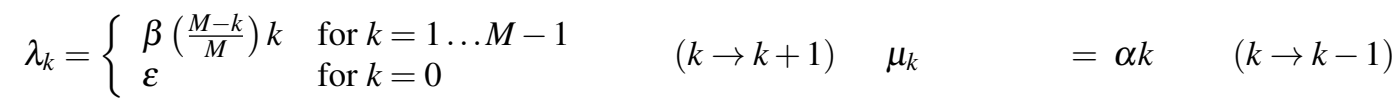

In this revision version, the external factor $\varepsilon>0$ is kept only for $\lambda_{0}$. Here $\alpha$ and $\beta$ are strictly positive parameters, and $\varepsilon$ is a non-negative parameter. We define $\theta_{0}, \theta_{1}^{[\varepsilon]}, \ldots, \theta_{M}^{[\varepsilon]}$ by

$$
\theta_{0}=1 \quad \theta_{i}^{[\varepsilon]}=\frac{\lambda_{0} \lambda_{1} \cdots \lambda_{i-1}}{\mu_{1} \mu_{2} \cdots \mu_{i}} \quad \text { for } i=1, \ldots, M .
$$

The equilibrium distribution of $P_{M, \varepsilon}$ is derived as follows:

$$
\pi_{M}^{[\varepsilon]}(i)= \begin{cases}\frac{\theta_{0}}{\theta_{0}+\sum_{j=0}^{M-1} \theta_{(M-j)}^{[\varepsilon]}} & i=0 \\ \frac{\theta_{i}^{[\varepsilon]}}{\theta_{0}+\sum_{j=0}^{M-1} \theta_{(M-j)}^{[\varepsilon]}} & i=1,2, \ldots, M\end{cases}
$$

Lemma 3.1. Assume $\beta>0, \alpha>0, \varepsilon>0, M>0$ and $R=\frac{\beta}{\alpha}$. We have :

$$
\theta_{0}=1 \quad \theta_{M-k}^{[\varepsilon]}=\frac{\varepsilon}{R \alpha(M-k)}\left(\frac{R}{M}\right)^{M-k} \frac{M !}{k !} \quad \text { for } k=0, \ldots, M-1
$$

\section{Proof:}




$$
\begin{aligned}
\theta_{M-k}^{[\varepsilon]} & =\frac{\lambda_{0} \lambda_{1} \cdots \lambda_{(M-k-1)}}{\mu_{1} \mu_{2} \cdots \mu_{(M-k)}}=\frac{\lambda_{0}}{\mu_{(M-k)}} \prod_{j=1}^{M-k-1} \frac{\lambda_{j}}{\mu_{j}}=\frac{\varepsilon}{\alpha(M-k)} \prod_{j=1}^{M-k-1}\left[\frac{\beta}{\alpha}\left(\frac{M-j}{M}\right)\right] \\
& =\frac{\varepsilon}{\alpha(M-k)} \prod_{j=1}^{M-k-1} R\left(\frac{M-j}{M}\right)=\frac{\varepsilon}{R \alpha(M-k)}\left(\frac{R}{M}\right)^{M-k} \frac{M !}{k !}
\end{aligned}
$$

\subsection{Properties of Poisson Distribution}

Some properties of Poisson distribution ( Collis-Bird(1963), Nzokem(2020)) will be stated with proof and the results will be applied in the next subsection.

Lemma 3.2. Suppose $X$ follows a Poisson distribution with parameter $\lambda$ and

$\mu(d)=E[X \mid X \leq d] \quad \forall d \in \mathbb{N}^{*}$ Then: $\mu(d)=\lambda \frac{g(d-1)}{g(d)}$

where

$g(d)=\sum_{i=0}^{d} \frac{\lambda^{i}}{i !}$ and $\lim _{d \rightarrow \infty} \frac{g(d-1)}{g(d)}=1$

\section{Proof:}

Let us define the following function $p(x, \lambda, d)=P(X=x \mid X \leq d)$ for $x=0, \ldots, d$

$p(x, \lambda, d)=P(X=x \mid X \leq d)=\frac{p(X=x)}{p(X \leq d)}=\frac{\frac{\lambda^{x}}{x !}}{\sum_{i=0}^{d} \frac{\lambda^{i}}{i !}}=\frac{\frac{\lambda^{x}}{x !}}{g(d)}=\frac{g(d-1)}{g(d)} p(x, \lambda, d-1)$

with

$\frac{g(d-1)}{g(d)}=\frac{\sum_{i=0}^{d-1} \frac{\lambda^{i}}{i !}}{\sum_{i=0}^{d} \frac{\lambda^{i}}{i !}}=1-\frac{\frac{\lambda^{d}}{d !}}{\sum_{i=0}^{d} \frac{\lambda^{i}}{i !}}$ and $\lim _{d \rightarrow \infty} \frac{g(d-1)}{g(d)}=1-\frac{\lim _{d \rightarrow \infty} \frac{\lambda^{d}}{d !}}{\lim _{d \rightarrow \infty} \sum_{i=0}^{d} \frac{\lambda^{i}}{i !}}=1$

We also have $\lambda \frac{d g}{d \lambda}(d)=\sum_{j=1}^{d} j \frac{\lambda^{j}}{j !}=\lambda g(d-1)$ and the result follows

$\mu(d)=E[X \mid X \leq d]=\sum_{j=0}^{d} j p(j, \lambda, d)=\frac{\sum_{j=1}^{d} j \frac{\lambda^{j}}{j !}}{g(d)}=\frac{\lambda \frac{d g}{d \lambda}(d)}{g(d)}=\lambda \frac{g(d-1)}{g(d)}$

Corollary 3.3. Assume $R>1, M>0$, and $X$ follows a Poisson distribution with parameter $\frac{M}{R}$. Then: $E\left[\frac{X}{M} \mid X<M\right]=$ $\frac{1}{R} \frac{g(M-2)}{g(M-1)}$ with $\lim _{M \rightarrow \infty} \frac{g(M-2)}{g(M-1)}=1$

\section{Proof:}

From lemma 3.2, $\lambda=\frac{M}{R}$ and $d=M-1$

$E\left[\frac{X}{M} \mid X<M\right]=E\left[\frac{X}{M} \mid X \leq M-1\right]=\frac{1}{M} E[X \mid X \leq M-1]=\frac{1}{M} \mu(M-1)=\frac{1}{R} \frac{g(M-2)}{g(M-1)}$

and $E\left[\frac{X}{M} \mid X<M\right]=\frac{1}{R} \frac{g(M-2)}{g(M-1)}$

Lemma 3.4. Assume $X$ follows a Poisson distribution with parameter $\lambda$ and let $a>\lambda$.

We have:

$P(X>a) \leq \mathrm{e}^{-\lambda+a-a \log \left(\frac{a}{\lambda}\right)}$

\section{Proof:}

$M(\theta)=\mathrm{e}^{\lambda\left(\mathrm{e}^{\theta}-1\right)}$ is the moment generating function of the Poisson distribution.

$M(\theta)=E\left(\mathrm{e}^{\theta X}\right)=\sum_{i=0}^{k \rightarrow \infty} \mathrm{e}^{k \theta} P(X=k)>\mathrm{e}^{a \theta} P(X>a)$ and $P(X>a)<\mathrm{e}^{\lambda\left(\mathrm{e}^{\theta}-1\right)-a \theta} \forall \theta \in \mathbb{R}$

Therefore, $P(X>a) \leq \inf _{\theta \in \mathbb{R}}\left\{\mathrm{e}^{\lambda\left(\mathrm{e}^{\theta}-1\right)-a \theta}\right\}=\mathrm{e}^{-\lambda+a-a \log \left(\frac{a}{\lambda}\right)}$. The function $\psi(\theta)=\mathrm{e}^{\lambda\left(\mathrm{e}^{\theta}-1\right)-a \theta}$ reaches its minimum at $\theta^{*}=\log \left(\frac{a}{\lambda}\right) \square$

Lemma 3.5. Assume $X$ follows a Poisson distribution with parameter $\lambda=\frac{M}{R}$.

Then $P\left(X>\frac{M}{R}+\delta M\right) \leq \mathrm{e}^{\frac{\phi(\delta R)}{R} M} \quad \forall \delta>0$ where $\phi()$ is a function and $\phi(\delta R)<0$ 


\section{Proof:}

Let us define $\phi(x)=x-(1+x) \log (1+x)$ and it can be shown that : $\phi(x)<0 \quad \forall x>0$

For $x=\delta R$, we have $\phi(\delta R)=\delta R-(1+\delta R) \log (1+\delta R)<0$

For $a=\frac{M}{R}+\delta M$ and $\lambda=\frac{M}{R}$, we apply Lemma 3.4

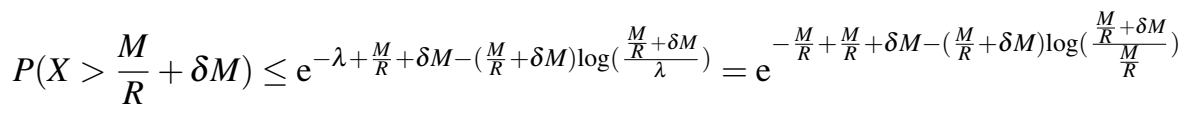

$$
\begin{aligned}
& \leq \mathrm{e}^{\frac{\phi(\delta R)}{R} M}
\end{aligned}
$$

\subsection{Limit Superior and Limit Inferior of the Expectation}

Lemma 3.6. Assume $R>1, M>0, X$ follows a Poisson distribution with parameter $\lambda=\frac{M}{R}$.

Then $\lim \sup _{M \rightarrow \infty} E\left(\frac{M}{M-X} \mid X<M\right) \leq \frac{1}{1-\frac{1}{R}}$

Proof: $I(X<M)$ is an indicator function,

$$
E\left[\frac{M}{M-X} I(X<M)\right]=E\left[\frac{M}{M-X} I(X \leq M *)\right]+E\left[\frac{M}{M-X} I(M *<X<M)\right]
$$

where $M *=\frac{M}{R}+\delta M$ and $\delta>0$. We assume that $M *<M$, which is equivalent to $0<\delta<\frac{R-1}{R}$

$$
E\left[\frac{M}{M-X} I(X \leq M *)\right] \leq \frac{1}{1-\frac{1}{R}-\delta} P\left(X \leq \frac{M}{R}+\delta M\right) \quad E\left[\frac{M}{M-X} I(M *<X<M)\right] \leq M P\left(\frac{M}{R}+\delta M<X\right)
$$

By applying Lemma 3.5 on (3.1), $E\left[\frac{M}{M-X} I(M *<X<M)\right] \leq M \mathrm{e}^{\frac{\phi(\delta R)}{R} M}$ where $\phi(\delta R)<0$.

$$
\begin{aligned}
E\left[\frac{M}{(M-X)} \mid X<M\right]=\frac{E\left[\frac{M}{M-X} I(X<M)\right]}{P(X<M)} & \leq \frac{P\left(X \leq \frac{M}{R}+\delta M\right)}{P(X<M)} \frac{1}{1-\frac{1}{R}-\delta}+\frac{1}{P(X<M)} M \mathrm{e}^{\frac{\phi(\delta R)}{R} M} \\
& \leq \frac{1}{1-\frac{1}{R}-\delta}+\frac{1}{P(X<M)} M \mathrm{e}^{\frac{\phi(\delta R)}{R} M}
\end{aligned}
$$

$\lim _{M \rightarrow \infty} \frac{1}{P(X<M)} M \mathrm{e}^{\frac{\phi(\delta R)}{R} M}=0$ and we have

$$
\begin{gathered}
\lim _{\delta \rightarrow 0} \limsup _{M \rightarrow \infty} E\left[\frac{M}{(M-X)} \mid X<M\right] \leq \lim _{\delta \rightarrow 0} \frac{1}{1-\frac{1}{R}-\delta} \quad \text { for } \quad 0<\delta<\frac{R-1}{R} \\
\limsup _{M \rightarrow \infty} E\left[\frac{M}{(M-X)} \mid X<M\right] \leq \frac{1}{1-\frac{1}{R}} \quad \square
\end{gathered}
$$

Lemma 3.7. Assume $R>1, M>0, X$ follows a Poisson distribution with parameter $\lambda=\frac{M}{R}$.

Then $\liminf _{M \rightarrow \infty} E\left(\frac{M}{M-X} \mid X<M\right) \geq \frac{1}{1-\frac{1}{R}}$

\section{Proof:}

The function $f_{M}(x)=\frac{M}{M-x}$ is convex over $0 \leq x<M$. Using the Jensen Inequality property,

$$
f_{M}(E[X \mid X<M]) \leq E\left[f_{M}(X) \mid X<M\right]
$$

From corollary 3.3, $E[X \mid X<M]=\frac{M}{R} \frac{g(M-2)}{g(M-1)}$

And from (3.2), we have

$$
E\left[\frac{M}{M-X} \mid X<M\right] \geq \frac{M}{M-E[X \mid X<M]}=\frac{M}{M-\frac{M}{R}\left(\frac{g(M-2)}{g(M-1)}\right)}
$$


We take the limit

$$
\liminf _{M \rightarrow \infty} E\left[\frac{M}{M-X} \mid X<M\right] \geq \liminf _{M \rightarrow \infty} \frac{1}{1-\frac{1}{R}\left(\frac{g(M-2)}{g(M-1)}\right)}=\frac{1}{1-\frac{1}{R}}
$$

From lemma 3.6 and lemma 3.7, we have:

$$
\lim _{M \rightarrow \infty} E\left[\frac{M}{M-X} \mid X<M\right]=\frac{1}{1-\frac{1}{R}}
$$

3.3 Approximation of the Asymptotic Distribution of $P_{M, \varepsilon}$

Lemma 3.8. Assume $\beta>0, \alpha>0, \varepsilon>0, M>0$ with $R=\frac{\beta}{\alpha}>1$.

$$
\sum_{k=0}^{M} \theta_{(M-k)}^{[\varepsilon]}=1+\sum_{k=0}^{M-1} \theta_{(M-k)}^{[\varepsilon]} \sim C(M) \frac{R}{R-1} \mathrm{e}^{\left(\frac{M}{R}\right)} \quad \text { As } M \rightarrow \infty \quad \text { and } C(M)=\frac{M ! \varepsilon}{M R \alpha}\left(\frac{R}{M}\right)^{M}
$$

\section{Proof:}

$$
\begin{aligned}
\sum_{k=0}^{M-1} \theta_{M-k}^{[\varepsilon]} & =\sum_{k=0}^{M-1} \frac{\varepsilon}{R \alpha(M-k)}\left(\frac{R}{M}\right)^{M-k} \frac{M !}{k !} \quad\left(\theta_{M-k}^{[\varepsilon]}\right. \text { from Lemma 3.1) } \\
& =C(M) \mathrm{e}^{\left(\frac{M}{R}\right)} \sum_{k=0}^{M-1} \frac{M}{M-k} \frac{1}{k !}\left(\frac{M}{R}\right)^{k} \mathrm{e}^{\left(-\frac{M}{R}\right)} \quad \text { with } C(M)=\frac{M ! \varepsilon}{M R \alpha}\left(\frac{R}{M}\right)^{M} \\
& =C(M) \mathrm{e}^{\left(\frac{M}{R}\right)} \sum_{k=0}^{M-1} \frac{M}{M-k} P[X=k] \quad \text { with } X \sim \text { Poisson }\left(\frac{M}{R}\right) \\
& =C(M) \mathrm{e}^{\left(\frac{M}{R}\right)} E\left[\frac{M}{M-X} I(X<M)\right] \quad(I(X<M) \text { indicator function })
\end{aligned}
$$

From the conditional expectation:

$$
E\left[\frac{M}{(M-X)} I(X<M)\right]=P[X<M] E\left[\frac{M}{M-X} \mid X<M\right]
$$

We deduce the following relation

$$
\frac{1+\sum_{k=0}^{M-1} \theta_{(M-k)}^{[\varepsilon]}}{C(M) \mathrm{e}^{\left(\frac{M}{R}\right)}\left(\frac{R}{R-1}\right)}=\left(1-\frac{1}{R}\right) E\left[\frac{M}{M-X} I(X<M)\right]+\frac{1}{C(M) \mathrm{e}^{\left(\frac{M}{R}\right)}\left(\frac{R}{R-1}\right)}
$$

From the results (3.4) and (3.3), we have

$$
\lim _{M \rightarrow \infty}\left(1-\frac{1}{R}\right) E\left[\frac{M}{M-X} I(X<M)\right]=1
$$

Nzokem(2020) shows that

$$
\lim _{M \rightarrow \infty} \frac{1}{C(M) \mathrm{e}^{\left(\frac{M}{R}\right)}\left(\frac{R}{R-1}\right)}=0 \quad \forall R>1
$$

From (3.5) and (3.6), we have :

$$
\lim _{M \rightarrow \infty} \frac{1+\sum_{k=0}^{M-1} \theta_{(M-k)}^{[\varepsilon]}}{C(M) \mathrm{e}^{\left(\frac{M}{R}\right)}\left(\frac{R}{R-1}\right)}=1
$$

Theorem 3.9. Assume $\beta>0, \alpha>0, \varepsilon>0, M>0$ with $R=\frac{\beta}{\alpha}>1$. The infected size has the following equilibrium distribution.

$$
\pi_{M}^{[\varepsilon]}(k) \sim \pi(k) \quad \text { As } M \rightarrow \infty
$$

with

$$
\pi(k)=\frac{R-1}{R} \frac{M}{(M-k) ! k}\left(\frac{M}{R}\right)^{M-k} \mathrm{e}^{\left(-\frac{M}{R}\right)} \quad(k=1,2, \ldots, M)
$$


Proof: For $k=1,2, \ldots, M$

$$
\begin{aligned}
\pi_{M}^{[\varepsilon]}(k) & =\frac{\theta_{k}^{[\varepsilon]}}{1+\sum_{j=0}^{M-1} \theta_{(M-j)}^{[\varepsilon]}}=\frac{1}{\frac{1+\sum_{j=0}^{M-1} \theta_{(M-j)}^{[\varepsilon]}}{C(M)\left(\frac{R}{R-1}\right) \mathrm{e}^{\left(\frac{M}{R}\right)}}} \frac{R-1}{R} \frac{M}{(M-k) ! k}\left(\frac{M}{R}\right)^{M-k} \mathrm{e}^{-\left(\frac{M}{R}\right)} \\
= & \frac{1}{\frac{1+\sum_{j=0}^{M-1} \theta_{(M-j)}^{[\varepsilon]}}{C(M)\left(\frac{R}{R-1}\right) \mathrm{e}^{\left(\frac{M}{R}\right)}}} \pi(k)
\end{aligned}
$$

We have the following quotient:

$$
\frac{\pi_{M}^{[\varepsilon]}(k)}{\pi(k)}=\frac{1}{\frac{1+\sum_{j=0}^{M-1} \theta_{(M-j)}^{[\varepsilon]}}{C(M)\left(\frac{R}{R-1}\right) \mathrm{e}^{\left(\frac{M}{R}\right)}}}
$$

The result follows from lemma $3.8 \square$

Theorem 3.10. Assume $\beta>0, \alpha>0, M>>1$ with $R=\frac{\beta}{\alpha}>1$. The infected size follows asymptotically a normal distribution with mean $\mu=\left(1-\frac{1}{R}\right) M$ and variance $\sigma^{2}=\frac{M}{R}$.

\section{Proof:}

We know that $(M-k) ! \sim \sqrt{2 \pi} \mathrm{e}^{-(M-k)}(M-k)^{(M-k)+\frac{1}{2}}$ for $M \rightarrow \infty$

$$
\begin{aligned}
\pi(k)=\frac{R-1}{R} \frac{M}{(M-k) ! k}\left(\frac{M}{R}\right)^{M-k} \mathrm{e}^{-\left(\frac{M}{R}\right)} & \sim \frac{R-1}{R \sqrt{2 \pi}} \frac{M}{k(M-k)^{\frac{1}{2}}}\left(\frac{M}{(M-k) R}\right)^{M-k} \mathrm{e}^{-\left(\frac{M}{R}\right)+(M-k)} \\
& =\psi(k)
\end{aligned}
$$

For $M \rightarrow \infty$, we have :

Supposed $x=k=\left(1-\frac{1}{R}\right) M(1+\delta)$.

$$
\pi(k) \sim \psi(k)
$$

By applying the second order Taylor's expansion techniques, Nzokem (2020) shows the following expression

$$
\begin{aligned}
\log (\psi(k))= & \log \left(\frac{R-1}{R \sqrt{2 \pi}}\right)+\log \left(\frac{M}{k}\right)-\frac{1}{2} \log (M-k)+(M-k) \log \left(\frac{M}{(M-k) R}\right)-\frac{M}{R}+(M-k) \\
=- & \frac{1}{2} \log \left(2 \pi \frac{M}{R}\right)-\frac{1}{2} \frac{(R-1)^{2}}{R} M \delta^{2}+\left[\frac{(R-3)}{2} \delta+\frac{1}{4}\left((R-1)^{2}+2\right) \delta^{2}+O_{1}\left(\delta^{3}\right)\right] \\
& +\left[-\frac{1}{2} \frac{(R-1)^{3}}{R} M \delta^{3}-\left(\frac{1-(R-1) \delta}{R} M+\frac{1}{2}\right) O_{2}\left(\delta^{3}\right)\right]
\end{aligned}
$$

We have the following expression

$$
\psi(k)=\frac{1}{\sqrt{2 \pi \frac{M}{R}}} \mathrm{e}^{\left\{-\frac{1}{2} \frac{(R-1)^{2}}{R} M \delta^{2}\right\}} \mathrm{e}^{\left\{\frac{(R-3)}{2} \delta+\frac{1}{4}\left((R-1)^{2}+2\right) \delta^{2}+O_{1}\left(\delta^{3}\right)\right\}} \mathrm{e}^{\left\{-\frac{1}{2} \frac{(R-1)^{3}}{R} M \delta^{3}-\left(\frac{1-(R-1) \delta}{R} M+\frac{1}{2}\right) O_{2}\left(\delta^{3}\right)\right\}}
$$

Now, we can prove the Local Central Limit Theorem. For $k$ integer, to get a convenient limit, we will choose $m_{M}, \sigma_{M}$ and $k$ as a function of $M(k=k(M))$ that satisfy the following properties:

$$
\lim _{M \rightarrow \infty} \frac{k-m_{M}}{\sigma_{M}}=s \quad \operatorname{Pr}\left(X_{M}=k(M)\right) \sim \frac{1}{\sqrt{2 \pi} \sigma_{M}} \mathrm{e}^{-s^{2} / 2} \quad \text { as } M \rightarrow \infty
$$

For theorem 3.10, we will choose $m_{M}=\left(1-\frac{1}{R}\right) M$ and $\sigma_{M}=\sqrt{M / R}$. Fix a real number $s$, we choose the sequence $k(M)$ such as

$$
\lim _{M \rightarrow \infty} \frac{k(M)-m_{M}}{\sigma_{M}}=s
$$


$k(M)$ is provided by the previous Taylor's expansion condition $k(M)=\left(1-\frac{1}{R}\right) M(1+\delta)$ with $\operatorname{Min}\left(\frac{1}{R-1}, 1\right)>\delta$. The limit (3.9) holds if $\delta=\delta(M)=\frac{s \sqrt{R}}{(R-1) \sqrt{M}}$. In addition, we have the following property

$$
\lim _{M \rightarrow \infty} \delta=0 \quad \lim _{M \rightarrow \infty} M \delta^{3}=\lim _{M \rightarrow \infty} \frac{s^{3} R^{\frac{3}{2}}}{(R-1)^{3} \sqrt{M}}=0
$$

From (3.7) and the function (3.8), as $M \rightarrow \infty$, we have:

$$
\pi(k) \sim \frac{1}{\sqrt{2 \pi} \sigma_{M}} \mathrm{e}^{\left\{-\frac{1}{2} s^{2}\right\}}
$$

\section{Conclusion}

The dynamics of the infected size and its distribution at the equilibrium were our main interests in the article. The stochastic simulations show that the infected size sample paths converge to a stationary stochastic process, which depends on the reproduction number $(R)$. When the reproduction number $(R)$ increases for $R>1$, the infected size sample path increases as a whole. The numerical results also show a bell-shaped density distribution and provide statistical evidence that the stationary distribution is symmetric with the same tails distribution as the normal distribution. The analytical results show that the stationary stochastic process has a normal distribution with mean $\left(1-\frac{1}{R}\right) M$ and Variance $\frac{M}{R}$ when $M$ becomes larger.

\section{Acknowledgement}

I would like to express my special thanks to Prof. Neal Madras for providing advice and feedback on this article.

\section{References}

Allen, L. J. (2008). An introduction to stochastic epidemic models. In Mathematical epidemiology (pp. 81-130). Springer, Berlin, Heidelberg. https://doi.org/10.1007/978-3-540-78911-6_3

Clancy, D., \& Mendy, S. T. (2011). Approximating the quasi-stationary distribution of the SIS model for endemic infection. Methodology \& Computing in Applied Probability, 13(3), 603-618. https://doi.org/10.1007/s11009-010-9177-8

Collis-Bird, J. (1963). The poisson distribution. PhD thesis, McGill University Libraries.

Karlin, S., \& Taylor, H. E. (1975). A first course in stochastic processes. New York : Academic Press. https://doi.org/10.1016/B978-0-08-057041-9.50005-2

Karlin, S., \& Taylor, H. M. (1998). An introduction to stochastic modeling. Academic Press, New York.

Näsell, I. (1996). The quasi-stationary distribution of the closed endemic SIS model. Advances in Applied Probability, 895-932. https://doi.org/10.1016/S0025-5564(98)10059-7

Näsell, I. (1999). On the quasi-stationary distribution of the stochastic logistic epidemic. Mathematical biosciences, 156(1-2), 21-40. https://doi.org/10.1016/S0025-5564(98)10059-7

Nzokem, A. H. (2020). Stochastic and renewal methods applied to epidemic models. PhD thesis, York University, YorkSpace institutional repository.

Nzokem, A., \& Madras, N. (2020). epidemic dynamics and adaptive vaccination strategy: renewal equation approach. Bulletin of Mathematical Biology, 82(9), 1-16. https://doi.org/10.1007/s11538-020-00802-2

Nzokem, A., \& Madras, N. (2021). Age-structured epidemic with adaptive vaccination strategy: Scalar-renewal equation approach. Recent Developments in Mathematical, Statistical and Computational Sciences. Springer.

Ovaskainen, O. (2001). The quasistationary distribution of the stochastic logistic model. Journal of Applied Probability, 38(4), 898-907. https://doi.org/10.1239/jap/1011994180

Wierman, J. C., \& Marchette, D. J. (2004). Modeling computer virus prevalence with a susceptible-infected-susceptible model with reintroduction. Computational statistics \& data analysis, 45(1), 3-23. https://doi.org/10.1016/S01679473(03)00113-0 


\section{Copyrights}

Copyright for this article is retained by the author(s), with first publication rights granted to the journal.

This is an open-access article distributed under the terms and conditions of the Creative Commons Attribution license (http://creativecommons.org/licenses/by/4.0/). 